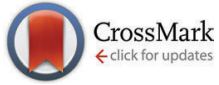

Cite this: Phys. Chem. Chem. Phys., $2016,18,17265$

Received 11th February 2016, Accepted 5th April 2016

DOI: $10.1039 / c 6 c p 00957 c$

www.rsc.org/pccp

\title{
The adsorbed state of a thiol on palladium nanoparticles
}

\author{
Scott M. Rogers, ${ }^{\text {ab }}$ Nikolaos Dimitratos, ${ }^{\text {ac }}$ Wilm Jones, ${ }^{\text {bc }}$ Michael Bowker, ${ }^{\text {bc }}$ \\ Antonios G. Kanaras, ${ }^{d}$ Peter P. Wells, ${ }^{\text {ab }}$ C. Richard A. Catlow ${ }^{a b c}$ and \\ Stewart F. Parker*be
}

\begin{abstract}
In the present work, a combination of imaging, spectroscopic and computational methods shows that 1-dodecanethiol undergoes $S$-deprotonation to form 1-dodecanethiolate on the surface of palladium nanoparticles, which then self-assembles into a structure that shows a high degree of order. The alkyl chain is largely in the all-trans conformation, which occurs despite the small size of the nanoparticle, (mean diameter $=3.9 \mathrm{~nm}$ ). Inelastic neutron scattering spectroscopy is readily able to characterise organic surface layers on nanoparticles; the nature of the material is irrelevant: whether the nanoparticle core is an oxide, a metal or a semiconductor makes no difference. Comparison to DFT calculations allows insights into the nature and conformation of the adsorbed layer.
\end{abstract}

\section{Introduction}

Nanoparticle size materials have attracted remarkable academic and industrial interest due to the high surface area to volume ratio. ${ }^{1,2}$ The main challenges of the academic and industrial researchers in the area of nanoparticles are the controllable syntheses of metal nanoparticles with a desired shape, size and structure. ${ }^{3}$ Solving these challenges will facilitate the commercial utilisation of nanoparticles in many areas ranging across chemical sensing, biolabeling, catalysis, photonics, and semiconductors. In the area of catalysis, the utilisation of supported metal nanoparticles (Au, Pd, Pt) has been shown to be particularly effective for a broad range of catalytic reactions such as oxidation, hydrogenation and combustion of volatile organic compounds (VOCs). ${ }^{4-9}$ These include the oxidation of $\mathrm{CO},{ }^{10,11}$ the selective oxidation of alcohols and polyols, ${ }^{12-14}$ the epoxidation of olefins, ${ }^{15,16}$ the hydrochlorination of ethyne, ${ }^{17,18}$ the selective hydrogenation of unsaturated carbonyl and nitro groups, ${ }^{19}$ and the direct synthesis of hydrogen peroxide from molecular hydrogen and oxygen. ${ }^{20,21}$ Typical methodologies for the synthesis of supported metal nanoparticles are based

\footnotetext{
${ }^{a}$ Department of Chemistry, University College London, 20 Gordon Street, London, WC1H OAJ, UK

${ }^{b}$ UK Catalysis Hub, Research Complex at Harwell, STFC Rutherford Appleton Laboratory, Chilton, Didcot, Oxfordshire OX11 OFA, UK

${ }^{c}$ Cardiff Catalysis Institute, School of Chemistry, Cardiff University, Cardiff, CF10 3AT, UK

${ }^{d}$ School of Physics and Astronomy, Faculty of Physical Sciences and Engineering, University of Southampton, Highfield, Southampton, SO17 1BJ, UK

${ }^{e}$ ISIS Facility, STFC Rutherford Appleton Laboratory, Chilton, Didcot, Oxfordshire OX11 OQX, UK. E-mail: stewart.parker@stfc.ac.uk
}

primarily on colloidal methods, and to a lesser extent impregnation methods. The main advantages of colloidal methods are (i) control of the particle size, shape and dispersity by tuning reaction conditions such as the metal salt-to-ligand ratio, concentration and nature of the reducing agent, solvent and temperature, (ii) the large variety of functionalised ligands that can be introduced and (iii) isolation, cleaning and redispersion of the particles in different solvents. However, some disadvantages and challenges remain such as; (i) the effective removal of ligands after the synthesis of metal nanoparticles, (ii) the excess use of solvents and (iii) the effective scale up production of nanoparticles using chemical liquid phase methods in industry.

Nano-sized metal colloids are usually synthesised by an in situ reduction method from a suitable metal precursor, by chemical reduction (with $\mathrm{NaBH}_{4}$ or alcohol), photoreduction, electrochemical reduction or thermal decomposition. ${ }^{2}$ Small molecular ligands, surfactants and polymers have been used to stabilise the so-formed metallic colloids. In some cases, metal colloids are stable over long periods even without coagulation in a solvent. However, for prolonged stability in a sol state, and in order to obtain a uniform, controlled particle size, use of a stabilising ligand is essential. Typically, polymers such as polyvinyl alcohol, ${ }^{22,23}$ polyvinylpyrrolidone ${ }^{24-26}$ and ligands such as alkanethiols ${ }^{27-30}$ and alkylamines ${ }^{31}$ are employed. Among these systems, alkanethiolate-capped nanoparticles are considered as a well-defined system and many examples in the area of catalysis have shown the potential applications of Pd alkanethiolate-capped nanoparticles. However, because of their high ligand-surface coverage, they are not yet the most efficient catalytic materials for organic reactions, although a few examples have shown promising 
catalytic results such as $\mathrm{C}-\mathrm{C}$-coupling reactions, ${ }^{32}$ hydrogenation reactions, ${ }^{33}$ oxidation reactions ${ }^{34-36}$ and the isomerisation of allyl alcohols. $^{37,38}$

To understand and control the effect of alkanethiolate ligands on the catalytic properties of nanoparticles, it is essential, therefore to know the structure and conformation of the alkanethiol ligand. Surface ligands play a critical role in catalysis because they determine the accessibility of the reactant to the nanoparticle surface. In this paper we present our results using a combination of techniques including inelastic neutron scattering (INS) spectroscopy. INS spectroscopy ${ }^{39-41}$ is an ideal technique for studying the interaction of a metal with ligands because the metal nanoparticle is essentially invisible to neutrons and therefore only the organic layer is visible. This approach builds on that used to characterise successfully the hydroxyl and water layers on PdO. ${ }^{42}$

\section{Experimental}

\section{Synthesis}

The synthesis employed in this study is based on the two-phase Brust-Schiffrin method ${ }^{27}$ that has been popularly used for the synthesis of thiol-protected gold nanoparticles. The BrustSchiffrin method has been extensively documented and studied due to its ease of use and is still popular more than two decades after the first publication. ${ }^{43}$

Potassium tetrachloropalladate $\left(\mathrm{K}_{2} \mathrm{PdCl}_{4} ; 0.0188 \mathrm{~mol}\right)$ was dissolved in $0.627 \mathrm{~L}$ of Milli-Q water. Tetraoctylammonium bromide $(0.08356 \mathrm{~mol})$ was dissolved in $1.67 \mathrm{~L}$ of toluene. Both solutions were mixed and continuously stirred until the organic layer turned dark orange and the aqueous layer cleared, indicating the completion of the phase transfer of $\left[\mathrm{PdCl}_{4}\right]^{2-}$ to the organic layer, 1-dodecanethiol (3.55 g) was then added to the organic phase. Afterward, a freshly prepared aqueous solution of sodium borohydride $(0.4 \mathrm{M}, 0.517 \mathrm{~L})$ was slowly added to the vigorously stirred reaction mixture. A rapid color change to black was observed, indicating the formation of nanoparticles. Upon the completion of $8 \mathrm{~h}$ of continuous stirring, the aqueous layer was removed by using a separatory funnel and toluene was removed by vacuum. The resulting crude nanoparticles were washed by using $2 \mathrm{~L}$ of ethanol to remove excess thiol. The resulting nanoparticles were dried at room temperature. The preparation was repeated four times to produce $8 \mathrm{~g}$ of Pd nanoparticles.

\section{TEM-EDX}

Samples for examination by TEM were prepared by first dispersing $5 \mathrm{mg}$ of the Pd nanoparticles in high purity ethanol using ultra-sonication. $40 \mu \mathrm{L}$ of the black suspension was dropped on to a holey carbon film supported by a 300 mesh copper TEM grid before the solvent was evaporated. The sample was then examined using a JEOL JEM 2100 TEM model operating at $200 \mathrm{kV}$. Elemental analysis was performed using the mapping mode. Particle size analysis was carried out with Image J. ${ }^{44}$

\section{Inelastic neutron scattering (INS) spectroscopy}

INS spectroscopy was carried out on the high resolution broadband spectrometer TOSCA ${ }^{45}$ and the direct geometry spectrometer MAPS ${ }^{46}$ at the ISIS Pulsed Neutron and Muon Facility ${ }^{47}$ (Chilton, UK). The sample, $\sim 5 \mathrm{~g}$, was loaded into a thin-walled aluminium cell, which was inserted into the closed cycle refrigerator of the spectrometer and cooled to $<20 \mathrm{~K}$. The two spectrometers are complementary; for the present work the key features are that TOSCA provides good resolution spectra over $0-2000 \mathrm{~cm}^{-1}$ while MAPS enables the observation of the $\mathrm{S}-\mathrm{H}$ and $\mathrm{C}-\mathrm{H}$ stretch region. Raman spectra (785 $\mathrm{nm}$ excitation) were recorded simultaneously with the TOSCA INS spectra using a previously described system. ${ }^{48}$

\section{Computational studies}

A five layer palladium slab with (111) termination was generated from the bulk structure of palladium using the Materials Studio $^{49}$ package. $S$-Deprotonated 1-dodecanethiol was then added to one surface. A periodic system was created with a $30 \AA$ vacuum gap between layers. The large gap was to ensure that there was at least $10 \AA$ between the nearest atoms in different slabs. Two models of the thiol were investigated: one with the carbon backbone in the lowest energy all-trans configuration. For the second, 5000 molecular structures were generated via a molecular mechanics conformational search. This was done using the mixed Monte Carlo multiple minimization and Large Scale low mode method, as implemented in the MacroModel software (MacroModel v.10.3, Schrödinger). ${ }^{50}$ A high energy conformer with a significant number of gauche defects was then selected at random and used for the second configuration. Periodic density functional theory (periodicDFT) calculations were carried out using the plane wave pseudopotential method as implemented in the CASTEP code. ${ }^{51,52}$ Exchange and correlation were approximated using the PBE functional. The plane-wave cut-off energy was $940 \mathrm{eV}$. Brillouin zone sampling of electronic states was performed on a $8 \times 8 \times 1$ Monkhorst-Pack grid. The equilibrium structure, an essential prerequisite for lattice dynamics calculations was obtained by BFGS geometry optimization after which the residual forces were converged to zero within $\pm 0.008 \mathrm{eV} \AA^{-1}$. Phonon frequencies were obtained by diagonalisation of dynamical matrices computed using density-functional perturbation theory ${ }^{53}$ and also to compute the dielectric response and the Born effective charges. The structure with the all-trans dodecanethiol had $\mathrm{Cm}$ (no. 8) symmetry, while the gauche structure had $P 1$ (no. 1) symmetry. To make the phonon calculations more tractable for the latter, the constrained lattice dynamics method ${ }^{54}$ was used. In this case, all the Pd atoms except those in the top layer were "frozen", in essence, the atoms are assigned a mass of infinity and the corresponding entries of the dynamical matrix are set to zero. It is not necessary to perform any computations for perturbation of these atoms considerably reducing the computational resources needed. The atomic displacements in each mode that are part of the CASTEP output enable the visualization of the modes to aid assignments and are also all that is required to generate the INS 
spectrum using the program ACLIMAX ${ }^{55}$ We emphasise that for all the calculated spectra shown the transition energies have not been scaled.

\section{Results and discussion}

Fig. 1 shows a TEM image of the Pd nanoparticles and a histogram of the resulting particle size distribution. It can be seen that the synthesis has resulted in small particles with a uniform distribution. The EDX data, Fig. 2, confirm that sulfur is uniformly spread over the particles.
Fig. 3 shows the INS and Raman spectra in the S-H and $\mathrm{C}-\mathrm{H}$ stretch region. Adsorption of the 1-dodecanethiol, Fig. 3a, on the Pd nanoparticles, Fig. $3 \mathrm{~b}$, results in a $20 \mathrm{~cm}^{-1}$ blueshift, which is more clearly seen in the derivative-like difference spectrum, Fig. 3c. This also shows a negative-going peak at $2545 \mathrm{~cm}^{-1}$ (arrowed) that, by comparison to the Raman spectrum of solid 1-dodecanethiol, Fig. 3d, is assigned to the $\mathrm{S}-\mathrm{H}$ stretch. The peak is negative indicating loss of the thiol functionality, while the EDX shows the presence of sulfur, in combination the data show that 1-dodecanethiol, $\mathrm{C}_{12} \mathrm{H}_{25} \mathrm{SH}$, is present as a thiolate, $\mathrm{C}_{12} \mathrm{H}_{25} \mathrm{~S}$.
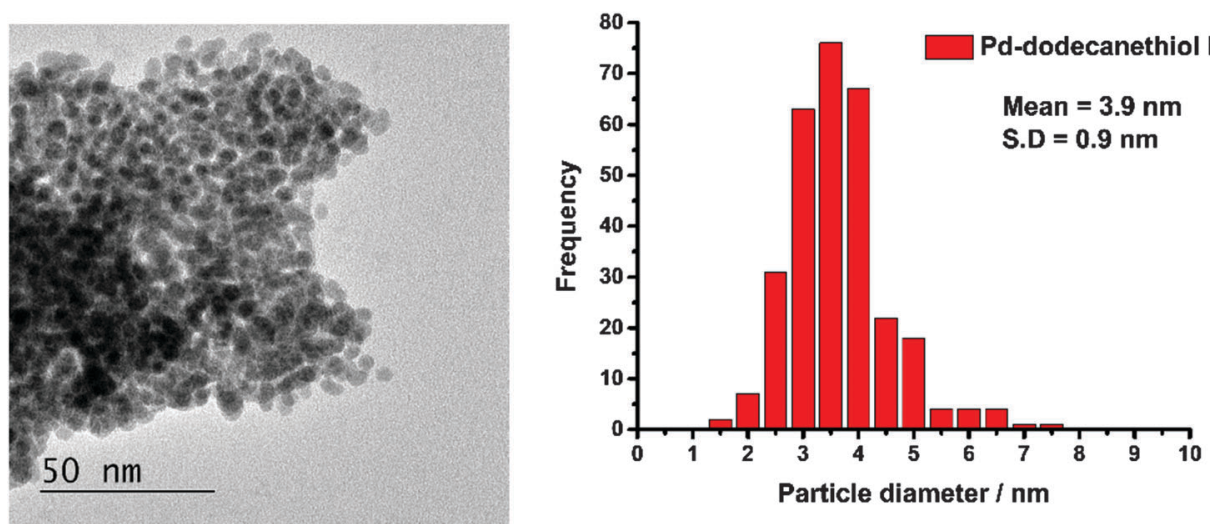

Fig. 1 Left: The TEM image of the 1-dodecanethiol coated Pd nanoparticles and right: the particle size distribution derived from the image.
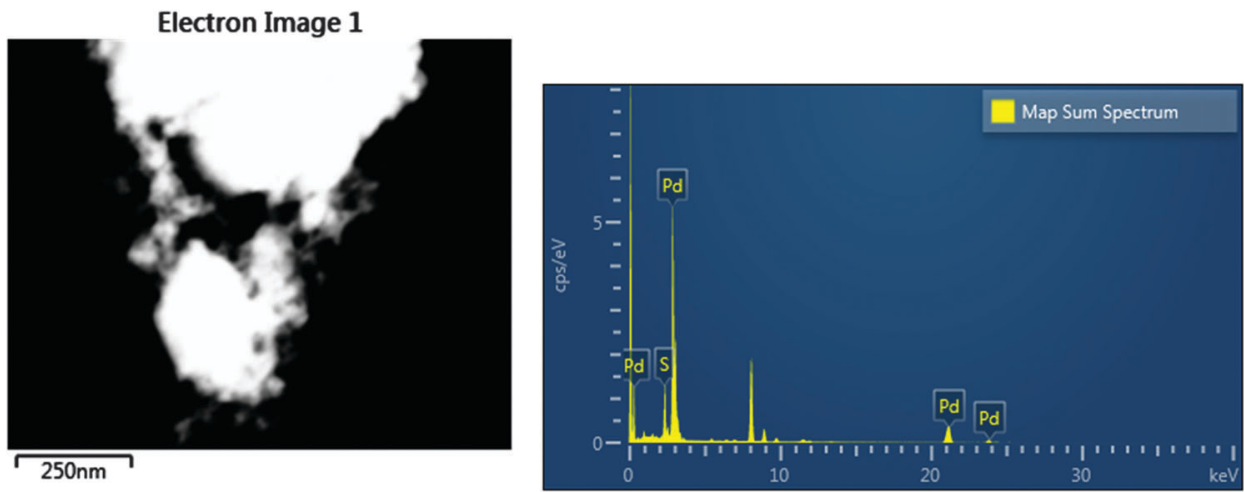

$\mathrm{Pd} K \alpha \mathbf{1}$

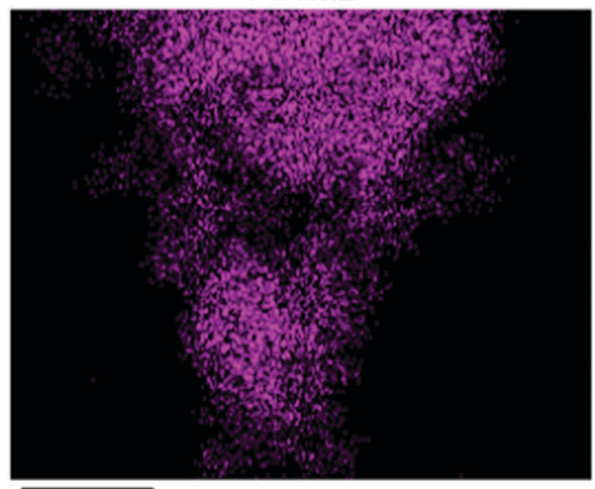

$250 \mathrm{~nm}$
$S \mathrm{~K} \alpha \mathbf{1}$

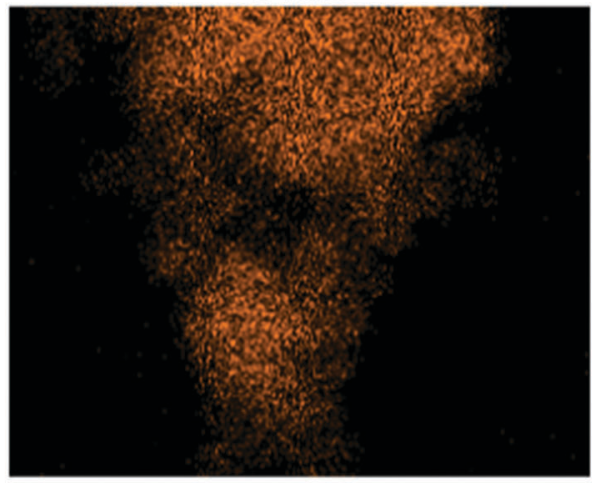

$\longdiv { 2 5 0 \mathrm { nm } }$

Fig. 2 Top left; The dark field STEM image, top right; the EDX spectrum, bottom; Pd and S Ka1 elemental intensity maps. 


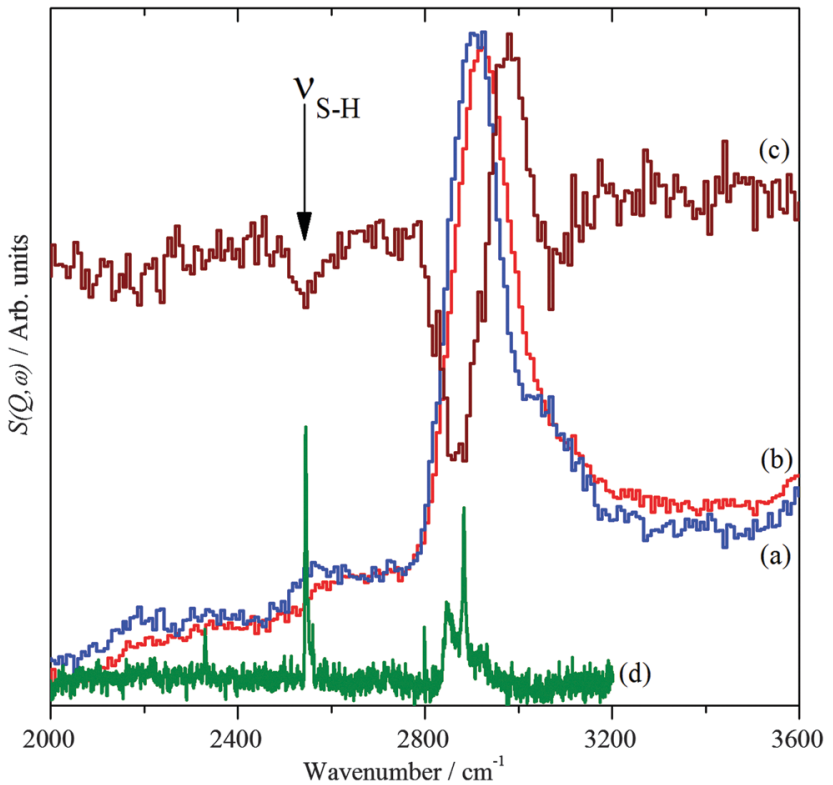

Fig. 3 INS spectra recorded on MAPS at $20 \mathrm{~K}$ of: (a) solid 1-dodecanethiol, (b) 1-dodecanethiol coated Pd nanoparticles and (c) the scaled difference spectrum ( $b-a),(c)$ is $\times 2$ ordinate expanded relative to (a). (d) The Raman spectrum of solid 1-dodecanethiol.

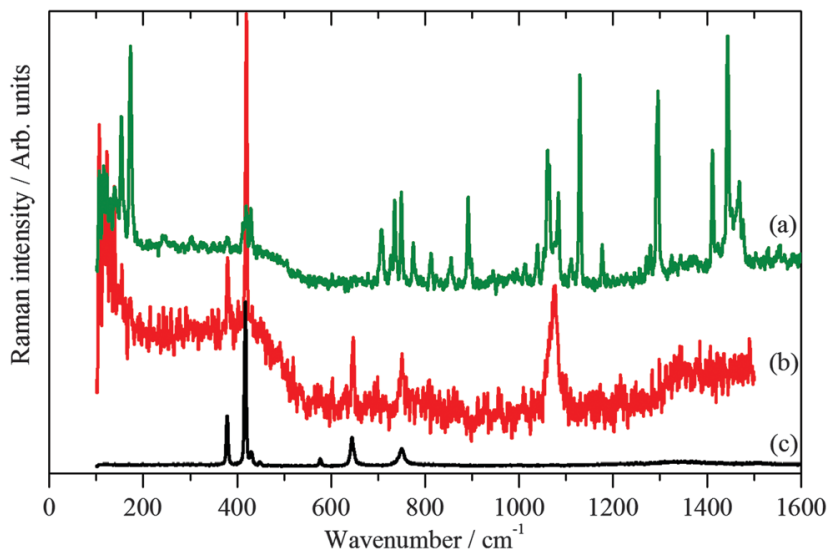

Fig. 4 Raman spectra at $20 \mathrm{~K}$ of: (a) solid 1-dodecanethiol and (b) 1-dodecanethiol coated Pd nanoparticles and (c) the sapphire window of the sample cup.

Fig. 4 and 5 show the Raman and INS spectra of solid 1-dodecanethiol and the coated Pd nanoparticles respectively. The Raman spectrum of the nanoparticles, Fig. $4 \mathrm{~b}$, only shows one band attributable to the thiolate at $1075 \mathrm{~cm}^{-1}$. In contrast the INS spectrum, Fig. 5b, shows many more features. Comparison with the INS spectrum of 1-dodecanethiol, Fig. 5a, shows both similarities and differences.

A useful similarity is that both INS spectra exhibit the $\mathrm{CH}_{2}$ rocking mode near $720 \mathrm{~cm}^{-1}$. This band occurs when there are sequences of methylene groups in the all-trans conformation and is diagnostic of them. ${ }^{56}$ For an infinite polymethylene $\left(\mathrm{CH}_{2}\right)_{n}$ chain the band occurs at $720 \mathrm{~cm}^{-1}$ and shifts to higher energy as $n$ decreases. For solid 1-dodecanethiol, the band occurs at $726 \mathrm{~cm}^{-1}$ and is at $728 \mathrm{~cm}^{-1}$ for the thiolate,



Fig. 5 INS spectra recorded on TOSCA at $20 \mathrm{~K}$ of: (a) solid 1-dodecanethiol and (b) 1-dodecanethiol coated Pd nanoparticles.

suggesting a shorter sequence of all-trans methylene groups in the adsorbed material, indicating some disorder in the chemisorbed layer.

In order to analyse Fig. 5 it is necessary to understand the spectra of the thiol. To our knowledge, the structure of 1-dodecanethiol has not been reported in any phase. However, the crystal structure of 1,12-dodecanedithiol is known. ${ }^{57}$ In the crystal there is no evidence for hydrogen bonding, thus the molecule can reasonably be treated as an isolated system. Accordingly, one of the thiol groups was replaced by a hydrogen atom to generate 1-dodecanethiol from 1,12-dodecanedithiol. Using the computational techniques, the structure was then geometry optimised and the vibrational spectrum calculated. A comparison of observed and calculated INS spectra is a stringent test of the model and as Fig. 6 shows the agreement is excellent. By setting the cross section of all the atoms to zero, except for the hydrogen of the thiol group, it is possible to isolate the modes that involve the motion of this group. In particular, it can be seen that there are prominent modes at 217,246 and $682 \mathrm{~cm}^{-1}$. Inspection of the mode visualisations shows that these are due to the out-of-plane $\mathrm{C}-\mathrm{S}-\mathrm{H}$ bend, the

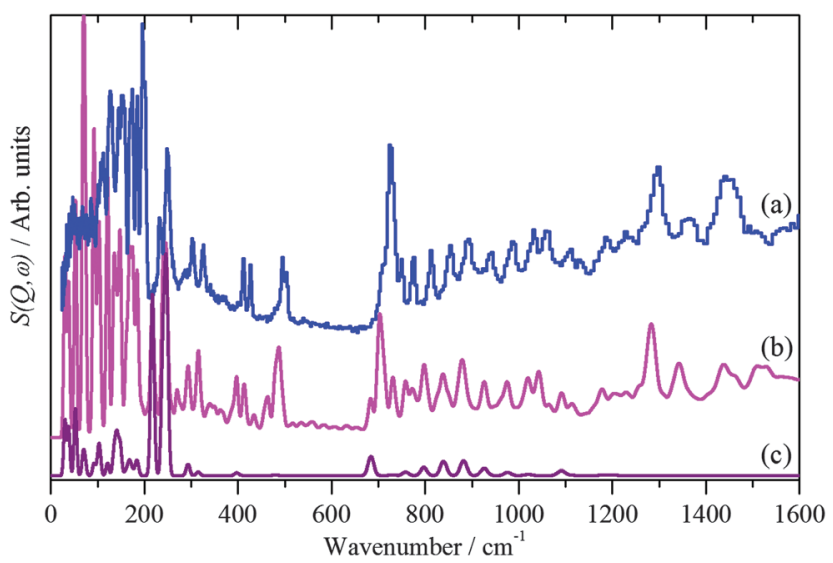

Fig. 6 INS spectra recorded of solid 1-dodecanethiol: (a) experimental, (b) calculated by CASTEP and (c) as (b) but only including the modes that involve motion of the thiol proton. 


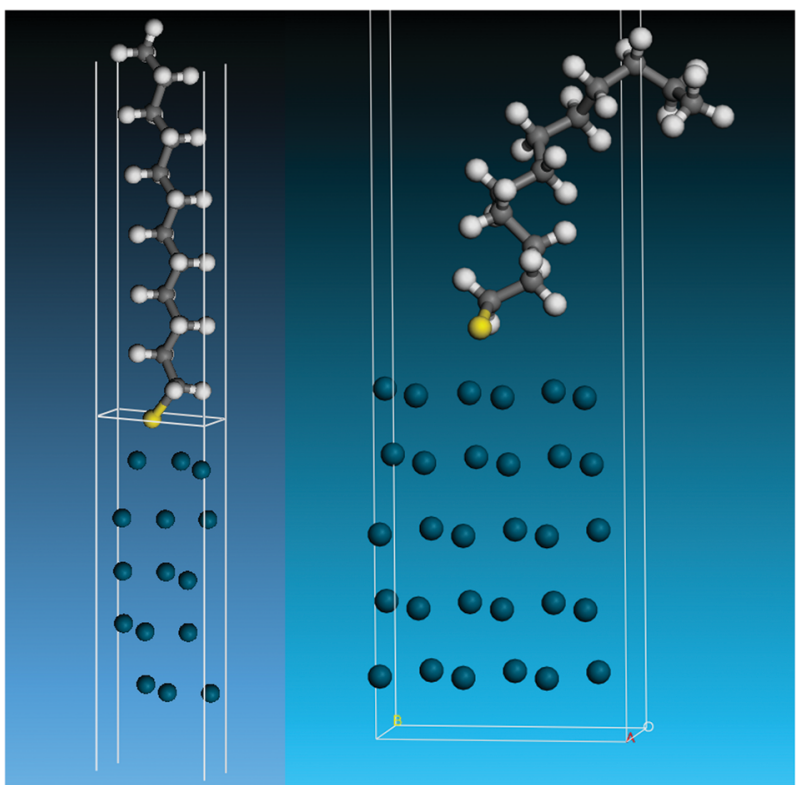

Fig. 7 Structures used to model the adsorbed thiolate on Pd(111). Left: All-trans thiolate. Right: Thiolate with gauche defects.

bend coupled to the methyl torsion and the in-plane $\mathrm{C}-\mathrm{S}-\mathrm{H}$ bend. The first and last of these are diagnostic of the thiol functionality and are observed in the experimental spectrum at 232 and $708 \mathrm{~cm}^{-1}$. Inspection of Fig. $5 \mathrm{~b}$ shows no trace of these modes, consistent with Fig. 3 and adsorption as thiolate. None of the spectra of the adsorbed species show any evidence for unreacted thiol, our experience is that this means that, at most, $\sim 10 \%$ of the adsorbate could be unreacted thiol.

To model the adsorbed thiolate, the anion was placed with the sulfur atom above the threefold site and the $\mathrm{C}-\mathrm{S}$ bond almost perpendicular to the surface, which resulted in a tilted structure with the carbon backbone at $\sim 30^{\circ}$ to the surface normal. The geometry optimised structure is shown in the left part of Fig. 7. It can be seen that the thiolate occupies a twofold bridge site $(\mathrm{C}-\mathrm{S}=2.291 \AA$ ) with the carbon backbone almost perpendicular to the surface. To model a disordered system, a thiolate with a large number of gauche defects was used. To prevent intermolecular contacts, it was necessary to use a doubled unit cell. This structure optimised to that shown on the right of Fig. 7.

The calculated INS spectra of the two structures are compared to the experimental data in Fig. 8. It can be seen that neither result exactly matches the data, although the all-trans conformation, Fig. 8b, is the closer match. In particular, in the 0-600 $\mathrm{cm}^{-1}$ region the in-plane $\mathrm{C}-\mathrm{C}-\mathrm{C}$ bending modes (longitudinal acoustic modes, LAMs) ${ }^{58}$ occur and overlap with the lower energy, $0-200 \mathrm{~cm}^{-1}$, out-of-plane $\mathrm{C}-\mathrm{C}-\mathrm{C}$ bending modes (transverse acoustic modes, TAMs). ${ }^{59}$ As can be seen from Fig. $8 \mathrm{~b}$ and $\mathrm{c}$, these modes are conformationally sensitive. The disordered chain predicts a sequence of almost constant intensity modes up to the bandhead at $510 \mathrm{~cm}^{-1}$, whereas the envelope of the ordered chain more closely matches the experimental profile. We also note that the disordered model does

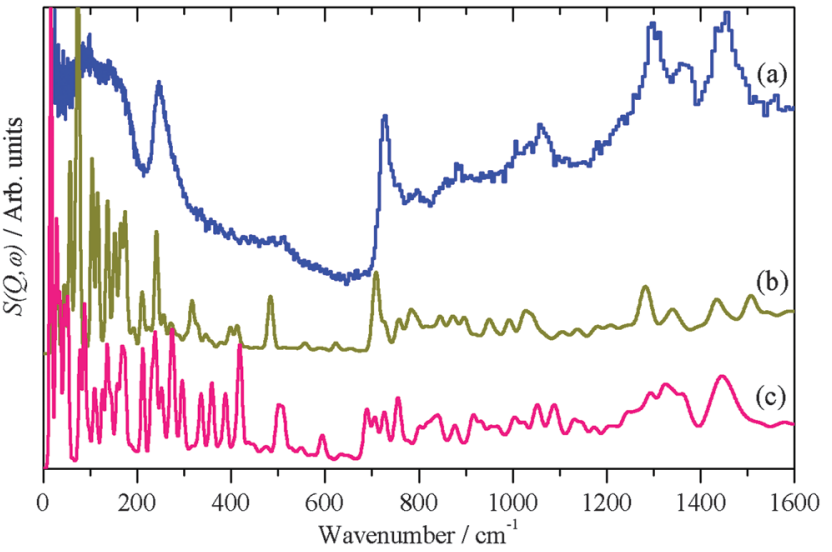

Fig. 8 (a) Experimental INS spectrum of thiolate on palladium nanoparticles and as calculated by CASTEP for thiolate on $\mathrm{Pd}(111)$ in the (b) all-trans conformation and (c) gauche conformation.

not reproduce the shape of the $\mathrm{CH}_{2}$ rocking mode at $728 \mathrm{~cm}^{-1}$. If the adsorbed thiolate was completely in the all-trans conformation, then individual LAM modes in the $200-500 \mathrm{~cm}^{-1}$ region would be visible, as seen in Fig. 5 a for the parent thiol. The disordered example used has only four gauche defects, yet this is sufficient to drastically alter the spectrum as compared to the all-trans arrangement, this indicates that there can only be one or two defects per chain. On balance, the modelling and the INS spectroscopy strongly suggest that the thiolate is largely, but not completely, ordered on the nanoparticle surface.

\section{Conclusions}

In the present work, a combination of imaging, spectroscopic and computational methods shows that 1-dodecanethiol undergoes $S$-deprotonation to form 1-dodecanethiolate on the surface of the nanoparticle, that then self-assembles into a structure that shows a high degree of order. The alkyl chain is largely in the all-trans conformation. This occurs despite the small size of the nanoparticle, (mean diameter $=3.9 \mathrm{~nm}$ ), even for a perfect nanocube there would only be $\sim 100$ molecules per face, the real material undoubtedly has fewer than this. The Hirshfeld analysis from the CASTEP results shows that there is a small positive charge ( +0.03 electron) on the hydrogens and a small negative charge $(-0.07$ electron) on the carbons, providing a very weak electrostatic attraction. The ordered structure maximises this attraction which provides a driving force for the order.

In this paper, we have shown that INS spectroscopy is readily able to characterise organic surface layers on nanoparticles; the nature of the material is irrelevant: whether the nanoparticle core is an oxide, a metal or a semiconductor makes no difference. This is because the scattered intensity depends on the inelastic cross section, which is independent of the electronic state of the atom. Since the scattering is dominated by modes involving hydrogen motion, provided that the nanoparticle core is actually (or almost) non-hydrogenous, the core 
will be invisible to INS spectroscopy. The capability to view just the adlayer is a major advantage of INS spectroscopy that is under-exploited. Comparison to DFT calculations allows insights into the nature and conformation of the adsorbed layer.

\section{Acknowledgements}

The UK Catalysis Hub is kindly thanked for resources and support provided via our membership of the UK Catalysis Hub Consortium and funded by EPSRC (grants EP/I038748/1, EP/I019693/1， EP/K014706/1， EP/K014668/1， EP/K014854/1, $\mathrm{EP} / \mathrm{K} 014714 / 1$ and EP/M013219/1). This research has been performed with the use of facilities at the Research Complex at Harwell. The authors would like to thank the Research Complex for access and support to these facilities and equipment. The STFC Rutherford Appleton Laboratory is thanked for access to neutron beam facilities. Computing resources (time on the SCARF compute cluster for the CASTEP calculations) were provided by STFC's e-Science facility. We would like to thank Dr Svemir Rudic (ISIS Facility, UK) for help with the MacroModel software.

\section{References}

1 A. Roucoux, J. Schulz and H. Patin, Chem. Rev., 2002, 102, 3757-3778.

2 M. C. Daniel and D. Astruc, Chem. Rev., 2004, 104, 293-346.

3 Y. N. Xia, Y. J. Xiong, B. Lim and S. E. Skrabalak, Angew. Chem., Int. Ed., 2009, 48, 60-103.

4 G. C. Bond and D. T. Thompson, Catal. Rev.: Sci. Eng., 1999, 41, 319-388.

5 T. Mallat and A. Baiker, Chem. Rev., 2004, 104, 3037-3058.

6 C. Della Pina, E. Falletta, L. Prati and M. Rossi, Chem. Soc. Rev., 2008, 37, 2077-2095.

7 N. Dimitratos, J. A. Lopez-Sanchez and G. J. Hutchings, Chem. Sci., 2012, 3, 20-44.

8 M. Sankar, N. Dimitratos, P. J. Miedziak, P. P. Wells, C. J. Kiely and G. J. Hutchings, Chem. Soc. Rev., 2012, 41, 8099-8139.

9 A. S. K. Hashmi and G. J. Hutchings, Angew. Chem., Int. Ed., 2006, 45, 7896-7936.

10 T. Akita, M. Kohyama and M. Haruta, Acc. Chem. Res., 2013, 46, 1773-1782.

11 A. A. Herzing, C. J. Kiely, A. F. Carley, P. Landon and G. J. Hutchings, Science, 2008, 321, 1331-1335.

12 A. Abad, P. Concepcion, A. Corma and H. Garcia, Angew. Chem., Int. Ed., 2005, 44, 4066-4069.

13 L. Prati and A. Villa, Acc. Chem. Res., 2014, 47, 855-863.

14 A. Villa, N. Dimitratos, C. E. Chan-Thaw, C. Hammond, L. Prati and G. J. Hutchings, Acc. Chem. Res., 2015, 48, 1403-1412.

15 A. K. Sinha, S. Seelan, S. Tsubota and M. Haruta, Angew. Chem., Int. Ed., 2004, 43, 1546-1548.

16 M. D. Hughes, Y. J. Xu, P. Jenkins, P. McMorn, P. Landon, D. I. Enache, A. F. Carley, G. A. Attard, G. J. Hutchings,
F. King, E. H. Stitt, P. Johnston, K. Griffin and C. J. Kiely, Nature, 2005, 437, 1132-1135.

17 B. Nkosi, M. D. Adams, N. J. Coville and G. J. Hutchings, J. Catal., 1991, 128, 378-386.

18 P. Johnston, N. Carthey and G. J. Hutchings, J. Am. Chem. Soc., 2015, 137, 14548-14557.

19 A. Corma and P. Serna, Science, 2006, 313, 332-334.

20 P. Landon, P. J. Collier, A. J. Papworth, C. J. Kiely and G. J. Hutchings, Chem. Commun., 2002, 2058-2059.

21 J. K. Edwards, E. Ntainjua, A. F. Carley, A. A. Herzing, C. J. Kiely and G. J. Hutchings, Angew. Chem., Int. Ed., 2009, 48, 8512-8515.

22 A. Villa, D. Wang, N. Dimitratos, D. S. Su, V. Trevisan and L. Prati, Catal. Today, 2010, 150, 8-15.

23 G. L. Brett, P. J. Miedziak, N. Dimitratos, J. A. LopezSanchez, N. F. Dummer, R. Tiruvalam, C. J. Kiely, D. W. Knight, S. H. Taylor, D. J. Morgan, A. F. Carleya and G. J. Hutchings, Catal.: Sci. Technol., 2012, 2, 97-104.

24 S. Albonetti, T. Pasini, A. Lolli, M. Blosi, M. Piccinini, N. Dimitratos, J. A. Lopez-Sanchez, D. J. Morgan, A. F. Carley, G. J. Hutchings and F. Cavani, Catal. Today, 2012, 195, 120-126.

25 R. Narayanan and M. A. El-Sayed, J. Am. Chem. Soc., 2003, 125, 8340-8347.

26 H. Tsunoyama, H. Sakurai, Y. Negishi and T. Tsukuda, J. Am. Chem. Soc., 2005, 127, 9374-9375.

27 M. Brust, M. Walker, D. Bethell, D. J. Schiffrin and R. Whyman, J. Chem. Soc., Chem. Commun., 1994, 801-802. 28 M. Brust, J. Fink, D. Bethell, D. J. Schiffrin and C. Kiely, J. Chem. Soc., Chem. Commun., 1995, 1655-1656.

29 I. Hussain, Z. X. Wang, A. I. Cooper and M. Brust, Langmuir, 2006, 22, 2938-2941.

30 C. K. Yee, R. Jordan, A. Ulman, H. White, A. King, M. Rafailovich and J. Sokolov, Langmuir, 1999, 15, 3486-3491.

31 M. Schulz-Dobrick, K. V. Sarathy and M. Jansen, J. Am. Chem. Soc., 2005, 127, 12816-12817.

32 F. Lu, J. Ruiz and D. Astruc, Tetrahedron Lett., 2004, 45, 9443-9445.

33 M. Biswas, E. Dinda, M. H. Rashid and T. K. Mandal, J. Colloid Interface Sci., 2012, 368, 77-85.

34 J. Llorca, M. Dominguez, C. Ledesma, R. J. Chimentao, F. Medina, J. Sueiras, I. Angurell, M. Seco and O. Rossell, J. Catal., 2008, 258, 187-198.

35 Y. Tai, W. Yamaguchi, K. Tajiri and H. Kageyama, Appl. Catal., A, 2009, 364, 143-149.

36 Y. Tai, W. Yamaguchi, M. Okada, F. Ohashi, K. Shimizu, A. Satsuma, K. Tajiri and H. Kageyama, J. Catal., 2010, 270, 234-241.

37 D. J. Gavia and Y. S. Shon, Langmuir, 2012, 28, 14502-14508. 38 D. J. Gavia, J. Koeppen, E. Sadeghmoghaddam and Y. S. Shon, RSC Adv., 2013, 3, 13642-13645.

39 N. G. Hamilton, I. P. Silverwood, R. Warringham, J. Kapitan, L. Hecht, P. B. Webb, R. P. Tooze, S. F. Parker and D. Lennon, Angew. Chem., Int. Ed., 2013, 52, 5608-5611.

40 D. Lennon and S. F. Parker, Acc. Chem. Res., 2014, 47, 1220-1227. 
41 I. P. Silverwood, S. M. Rogers, S. K. Callear, S. F. Parker and C. R. A. Catlow, Chem. Commun., 2016, 52, 533-536.

42 S. F. Parker, K. Refson, A. C. Hannon, E. R. Barney, S. J. Robertson and P. Albers, J. Phys. Chem. C, 2010, 114, 14164-14172.

43 R. Sardar, A. M. Funston, P. Mulvaney and R. W. Murray, Langmuir, 2009, 25, 13840-13851.

44 W. S. Rasband, ImageJ, U. S. National Institutes of Health, Bethesda, Maryland, USA, http://imagej.nih.gov/ij/ 1997-2015.

45 S. F. Parker, F. Fernandez-Alonso, A. J. Ramirez-Cuesta, J. Tomkinson, S. Rudic, R. S. Pinna, G. Gorini and J. Fernández Castañon, J. Phys.: Conf. Ser., 2014, 554, 012003.

46 S. F. Parker, D. Lennon and P. W. Albers, Appl. Spectrosc., 2011, 65, 1325-1341.

47 http://www.isis.stfc.ac.uk/.

48 M. A. Adams, S. F. Parker, F. Fernandez-Alonso, D. J. Cutler, C. Hodges and A. King, Appl. Spectrosc., 2009, 63, 727-732.

49 http://accelrys.com/products/collaborative-science/bioviamaterials-studio/.

50 Schrödinger Release 2014-1: MacroModel, version 10.3, Schrödinger, LLC, New York, NY, 2014.
51 S. J. Clark, M. D. Segall, C. J. Pickard, P. J. Hasnip, M. J. Probert, K. Refson and M. C. Payne, Z. Kristallogr., 2005, 220, 567.

52 K. Refson, P. R. Tulip and S. J. Clark, Phys. Rev. B: Condens. Matter Mater. Phys., 2006, 73, 155114.

53 V. Milman, A. Perlov, K. Refson, S. J. Clark, J. Gavartin and B. Winkler, J. Phys.: Condens. Matter, 2009, 21, 485404.

54 K. Refson, Phonons and Related Calculations in CASTEP, http://www.castep.org/.

55 A. J. Ramirez-Cuesta, Comput. Phys. Commun., 2004, 157, 226.

56 D. Lin-Vien, N. B. Colthup, W. G. Fateley and J. G. Grasselli, The Handbook of Infrared and Raman Characteristic Frequencies of Organic Molecules, Academic Press, New York, 1991.

57 N. Nakamura, K. Uno and Y. Ogawa, Acta Crystallogr., Sect. E: Struct. Rep. Online, 2001, 57, 0508.

58 D. A. Braden, S. F. Parker, J. Tomkinson and B. S. Hudson, J. Chem. Phys., 1999, 111, 429-437.

59 J. Tomkinson, S. F. Parker, D. A. Braden and B. S. Hudson, Phys. Chem. Chem. Phys., 2002, 4, 716-721. 